\title{
Mise en ouvre de la méthode de cartographie du ruissellement IRIP pour l'analyse des risques lies aux écoulements sur l'infrastructure ferroviaire
}

\author{
Judicaël DEHOTIN ${ }^{1}$, Blandine CHAZELLE ${ }^{1}$, Guillaume LAVERNE ${ }^{1}$, Ahlem HASNAOUI ${ }^{1}$, Lucie LAMBERT², \\ Pascal BREIL ${ }^{3}$, Isabelle BRAUD ${ }^{3}$
}

\author{
1. Direction I\&P Ingénierie et Projets, SNCF RESEAU, Ingénierie Technique, 6 avenue François Mitterrand 93574 LA PLAINE ST DENIS - \\ blandine.chazelle@sncf.fr; judicael.dehotin@sncf.fr \\ 2. Direction Générale Île de France - Direction des Projets Franciliens - Agence Grand Paris - Cap Lendit 1-7 place des Étoiles \\ 93213 La Plaine St Denis-lucie.lambert @sncf.fr \\ 3. Irstea, UR HHLY, Hydrologie-Hydraulique, 5 Rue de la Doua, CS 70077,69626VILLEURBANNE Cedex. pascal.breil@irstea.fr; \\ isabelle.braud@irstea.fr
}

\begin{abstract}
RÉSUMÉ. - Les voies ferrées sont des éléments structurant des bassins versants qui modifient les chemins de l'eau. Le réseau ferré national est équipé de dispositifs hydrauliques de collecte et d'acheminement des écoulements depuis l'amont de l'infrastructure jusqu'à un exutoire. Le ruissellement est à l'origine de nombreux incidents sur les infrastructures ferroviaires; autant sur les ouvrages linéaires que sur les aménagements connexes nécessaires à l'exploitation ferroviaire. Ces incidents peuvent trouver leur origine dans l'absence ou le mauvais fonctionnement des dispositifs hydrauliques dont le bassin versant a pu être mal apprécié ou a évolué en raison de modifications de l'occupation des sols. L'érosion de l'assise des voies de chemin de fer, leur submersion par les eaux ou la création d'obstacles par les coulées de boues sont les principaux dysfonctionnements pouvant avoir comme impacts des ralentissements, des interruptions des circulations ferroviaires, voire dans des cas plus rares, des déraillements. Irstea a mis au point une méthode de cartographie du ruissellement (IRIP- Inondation par Ruissellement Intense Pluvial) qui permet d'avoir une vision globale du ruissellement et de sa dynamique potentielle depuis les zones sources jusqu'à l'aval, au niveau des zones d'accumulation. Sur la base de cette méthodologie et en collaboration avec Irstea, la direction de l'Ingénierie de la SNCF développe un logiciel dans l'optique de mieux répondre aux besoins de diagnostic et de gestion des risques liés au ruissellement sur le réseau ferré national. Des travaux de validation de la méthode et son intégration dans le contexte ferroviaire sont en cours avec des données d'incidents liés au ruissellement, recensés par le gestionnaire d'infrastructure.
\end{abstract}

Mots-clefs : ruissellement, érosion, inondation, risques, IRIP, ouvrages linéaires, voies ferrées, infrastructure

\section{Applying runoff mapping method IRIP for flooding risk analysis on railway infrastructure}

\begin{abstract}
Railways can modify flow paths and have an impact on runoff transfer within catchments. The French railway network includes various structures designed to collect and safely route water flows from upstream of the railway infrastructures towards an outlet. Runoff is a risk for railway infrastructures and is source of many incidents impacting both the civil infrastructure but also all the secondary services required for the railway functioning. These risks occur due to dysfunction of hydraulic structures, which may have been inappropriately designed or for which the environment may have evolved due to land cover change. Erosion of the railway basement, flooding and mudslides are the most frequent consequences of a poorly functioning drainage network. Their impacts on rail operations can be: speed limitations, interruption of the traffic, and even derailments in the worst cases. Irstea developed a methodology for the runoff risk mapping called IRIP (Flooding by Intense Pluvial Runoff) which provides a vision at the catchment scale, of the surface runoff and its potential dynamics from the production areas down to the downstream areas of accumulation. Based on this methodology, the engineering department at SNCF, in collaboration with Irstea, is developing an operational tool, witch aims to improve the diagnosis and the management of runoff risk on the French railway network. An assessment of the method is presented, based on a data base collecting historical incidents relating to runoff.
\end{abstract}

Key-words: runoff, erosion, flooding, risk, IRIP method, linear feature, railway infrastructure

\section{INTRODUCTION}

Le présent article fait suite à une récente publication [Chazelle et al., 2012] qui pointait les enjeux de l'évaluation, de l'analyse et de la gestion des risques liés à l'eau pour le patrimoine ferroviaire existant. Le travail présenté ici s'insère dans une démarche plus vaste d'amélioration continue du management des risques naturels pour améliorer la sécurité et la régularité des lignes. Cette démarche consiste à capitaliser et à faire évoluer un savoir-faire historique de l'entreprise SNCF en matière de prise en compte des risques liés à l'eau. En tant que futur acteur du gestionnaire 
d'infrastructure unifié pour le ferroviaire, SNCF œuvre pour une meilleure gestion intégrée des risques naturels au sein du système ferroviaire.

L'implantation du réseau ferré est fortement contrainte par la géométrie de son profil en long et de son profil en travers $\mathrm{du}$ fait des capacités de traction et de freinage des convois de trains. Les aménagements de type déblai, remblai, tunnels ou viaducs sont créés pour s'affranchir des variations de la topographie existante [Chazelle et al., 2012]. Ces aménagements croisent des cours d'eau de grands bassins versants et des axes de ruissellement issus de très nombreux petits bassins versants. Ainsi les voies ferrées peuvent créer des barrières, ou des cheminements privilégiés pour les écoulements de surface.

Le ruissellement sur de petits bassins versants est à l'origine de nombreux dysfonctionnements sur l'infrastructure ferroviaire. Ces derniers peuvent conduire à des désordres voire des incidents au droit de l'infrastructure linéaire et des aménagements associés (gares, ateliers de maintenance, etc.) en fonction de l'événement climatique et de la vulnérabilité du site au ruissellement. Une meilleure compréhension de ce phénomène et l'anticipation de ses impacts potentiels sur le réseau contribue à une gestion durable et soutenable de la sécurité et de la régularité des circulations ferroviaires. Dans une démarche d'anticipation des incidents liés au ruissellement, la SNCF a développé des méthodes d'analyse des risques. Une bonne évaluation et localisation des apports des eaux de ruissellement le long du linéaire ferroviaire conditionnent d'une part le bon diagnostic des incidents et, d'autre part, les réponses " hydrauliques " à apporter, que ce soit en termes d'ouvrage (leur type, leur dimensionnement ou leur localisation), d'entretien ou de surveillance. Cependant, la complexité du phénomène de ruissellement a constitué un frein dans le développement d'outils et de méthodes permettant de mieux caractériser ce processus sur des secteurs de grande ampleur. A ce jour, l'identification des secteurs les plus sensibles et l'évaluation des débits des axes d'écoulements situés sur les bassins non jaugés comportent d'importantes incertitudes. En effet les méthodes habituellement utilisées en ingénierie sont en limite d'application pour ces petits bassins. C'est le cas des méthodes Socose et Crupédix (Min. Agriculture 1980) ou encore de la méthode rationnelle qui requiert la connaissance du coefficient de ruissellement (Satin 2006). Par ailleurs, la cartographie du ruissellement et des sinistres liés au ruissellement est peu abordée dans la littérature scientifique, y compris dans le contexte ferroviaire. Les méthodes les plus proches de cet objectif ont été développées pour la gestion de l'érosion des terres agricoles (Le Bissonnais 1998 ; Langlois et Delahaye 2002 ; Le Gouée, et Delahaye 2008.). Le LRPC a initié une action de recherche (LRPC et CETE Normandie 2009) dont l'objectif est de développer une cartographie du risque ruissellement. Cette méthodologie est basée sur le recensement et la classification des thalwegs. Toutefois, cette méthode exclut les versants et ne permet pas de localiser les zones de production. De façon générale, les méthodes habituelles ne cartographient pas les axes de transfert et les zones d'accumulation de manière explicite.

La méthode de cartographie mise au point par Irstea (IRIP Indicateur du Ruissellement Intense Pluvial) permet d'identifier d'amont en aval les zones de production, les zones de transfert et les zones d'accumulation du ruissellement [Dehotin et Breil, 2011a]. Elle permet d'avoir une vision globale de la dynamique potentielle des écoulements de surface à l'échelle d'un bassin versant. Sur la base de cette méthode et en collaboration avec Irstea, la Direction Projets
Système Ingénierie de SNCF Infrastructure développe un logiciel de cartographie du ruissellement. Si les fonctionnalités de base de ce logiciel peuvent intéresser tous les acteurs concernées par la question du ruissellement, l'ingénierie technique vise des applications opérationnelles propres au contexte ferroviaire. L'objectif premier est d'améliorer les méthodes d'analyse de risques utilisées à ce jour à la SNCF pour l'anticipation des incidents liés au ruissellement. La cartographie du ruissellement par la méthode IRIP sera confrontée avec la vulnérabilité de l'infrastructure. Cette dernière dépend de sa géométrie et des dispositifs hydrauliques existants au droit des voies ferrées qui lui confèrent une certaine résilience aux écoulements naturels. Par ailleurs, les travaux de validation de la méthode IRIP initiés par Irstea se poursuivent en s'appuyant cette fois-ci sur des données recensées par le gestionnaire d'infrastructure ferroviaire et liées au ruissellement.

Le présent article a pour objectif d'illustrer la pertinence de l'intégration de la méthode IRIP dans le contexte ferroviaire dans le cadre de l'analyse des risques liés aux écoulements de surface. Après avoir rappelé les enjeux $\mathrm{du}$ ruissellement pour l'infrastructure ferroviaire ainsi que les principes de la méthode IRIP, nous allons présenter un exemple de mise en œuvre de cette méthode dans le contexte ferroviaire et les perspectives qu'elle ouvre pour la gestion des risques liés au ruissellement.

\section{LE RUISSELLEMENT DANS LE CONTEXTE FERROVIAIRE}

\section{II.1. Description de l'impact du ruissellement sur l'infrastructure ferroviaire}

De nombreux incidents sur le réseau ferré en France sont causés chaque année par des phénomènes liés à l'eau et en particulier aux écoulements de surface. La vulnérabilité de l'ouvrage ferroviaire tient surtout à la nature granulaire et non revêtue de l'assise ferroviaire, ce qui lui confère un caractère érodable [Chazelle et al., 2012]. L'érosion de l'assise de la voie (talus et/ou plateforme ferroviaire et/ou ballast, Figure 1), la submersion par les eaux, les coulées de boues avec ou sans dépôt de matériaux sur la voie et ses équipements (Figure 2) sont les principaux impacts du ruissellement sur l'infrastructure ferroviaire. L'inondation de la plateforme peut avoir pour conséquence de polluer le ballast par des dépôts de boues qui génèrent des déformations de nivellement de la voie ; de créer des shuntages de circuit de voie qui engendrent des dysfonctionnements de la signalisation. Dans les cas les plus graves, en cas de débits importants, les écoulements ont aussi la capacité d'entraîner le ballast et donc de déstabiliser la voie, d'éroder la piste, les talus et les remblais.

Les désordres importants sont souvent constatés lors des événements pluvieux exceptionnels mais aussi pour des événements pluvieux plus courants parfois inférieurs au décennal. Ils sont d'autant plus importants que les vitesses d'écoulement sont élevées. Dans un grand nombre de cas, les incidents sont dus au ruissellement rapide des nombreux petits bassins versants interceptés par le réseau ferré. Ils trouvent leur origine dans l'absence ou le mauvais fonctionnement des dispositifs hydrauliques dont le contexte a pu être mal apprécié dès l'origine (cas peu fréquent) ou a évolué (modification de l'occupation des sols sur les bassins versants, aménagements des cours d'eau, etc.). Ces désordres génèrent des perturbations de la circulation ferroviaire qui 


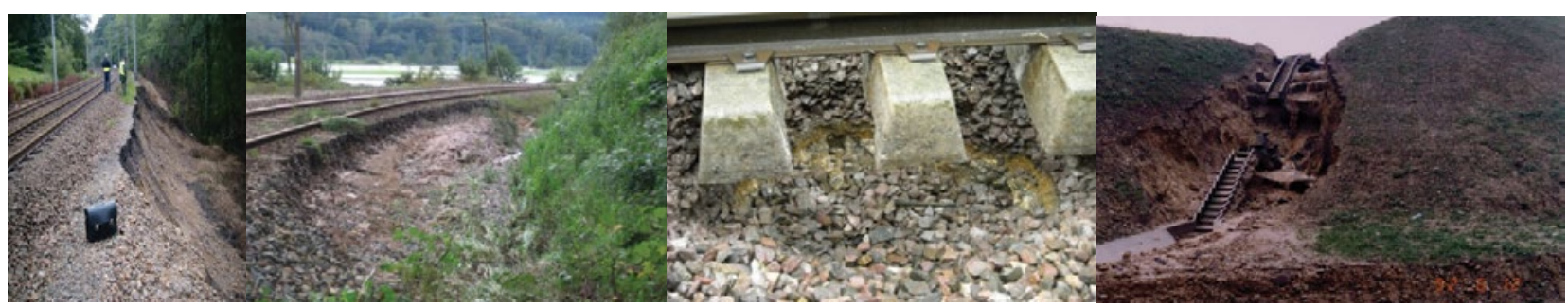

Figure 1 : Illustrations d'incidents graves : érosions de plateforme, de talus ou de remblais (Ingénierie SNCF Infra).

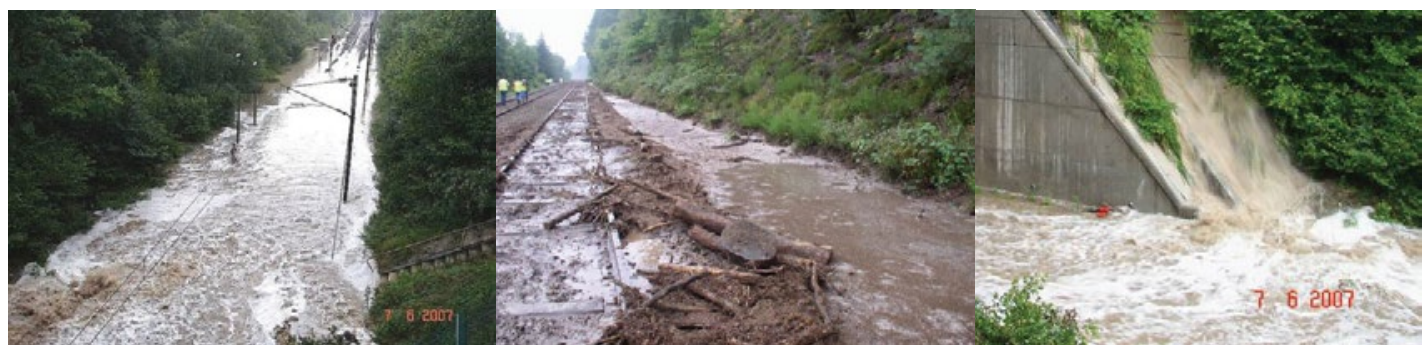

Figure 2 : Illustrations d'incidents graves : inondation et coulées boues (Ingénierie SNCF Infra).

sont classées par ordre de gravité de leur impact sur la sécurité et l'exploitation de la ligne :

- les incidents très graves (les déraillements et les heurts);

- l'arrêt complet des circulations sur la ligne ;

- l'arrêt des circulations sur la voie impactée par l'incident ;

- la mise en place de Limitation Temporaire de Vitesse (LTV).

\section{II.2. La gestion des risques ferroviaires liés au ruissellement : vers une anticipation des incidents}

Le réseau ferré a connu deux grandes périodes de développement. La première s'étend du début du $19^{\text {ème }}$ siècle avec la construction des premières lignes de transport de voyageurs et de marchandises jusqu'en 1914 et la première guerre mondiale. La seconde a débuté dans les années 1970 avec le développement du réseau à grande vitesse.

Les règles de conception ont évolué au cours des différentes phases de développement du réseau et intègrent à ce jour la notion de transparence hydraulique introduite par la Loi sur l'Eau [JORF, 1992, 2006] pour les nouvelles lignes. Cette notion est appliquée au cas par cas pour les travaux de régénération des lignes reconstruites à la suite d'une rupture d'ouvrage lors d'évènement exceptionnel [Pams Capoccioni $\&$ Cheetham, 2012]. En effet, le réseau ferré est équipé de dispositifs hydrauliques pour collecter et acheminer les écoulements depuis l'amont de l'infrastructure jusqu'à un exutoire à son aval. Il s'agit d'ouvrages linéaires le long de la voie ferrée (fossés, collecteurs drainants), d'ouvrages ponctuels situés sur les talus (descentes d'eau) ou d'ouvrages hydrauliques traversant la voie ferrée (aqueducs, dalots, portiques, viaducs etc.).

La capitalisation et les retours d'expérience sur le réseau ferré national sont organisés par des référentiels. « La connaissance des faits relatifs à la sécurité des circulations contribue au management de la sécurité et à la maîtrise du risque ferroviaire dans une démarche de sûreté de fonctionnement. Le retour d'expérience (REX) constitue l'un des outils majeurs de cette connaissance » (Référentiel de L'infrastructure ferroviaire Organisation du retour d'expérience risque ferroviaire). Ainsi, depuis 1998, une base de données historiques de ces incidents est constituée, animée et enrichie chaque année par la Direction I\&P Ingenierie et Projets de SNCF RESEAU.

Lorsque des incidents remettent en cause la sécurité des circulations, des mesures sont prises sur l'exploitation de la ligne : les circulations sont ralenties voire arrêtées selon l'ampleur des désordres en attendant des travaux de remise à niveau sur le tronçon de ligne impacté. Ces travaux peuvent alors être engagés en urgence pour permettre la remise en exploitation de la ligne dans les meilleurs délais. L'intervention peut prendre de quelques heures à plusieurs jours voire semaines dans les cas les plus graves.

La surveillance et l'alerte se heurtent encore à ce jour d'une part au manque de fiabilité de détection de potentiel de désordres et d'autre part aux difficultés d'intervention d'urgence. L'amélioration de l'identification des sites sensibles au ruissellement rapide et l'évaluation de l'importance de ces ruissellements, couplées à la prise en compte du rôle des dispositifs hydrauliques existants contribueraient à l'optimisation de la surveillance en ciblant mieux les interventions.

La gestion des risques liés à l'eau, commence lors du dimensionnement et de la conception, mais elle doit également prendre appui sur une politique d'entretien bien ciblée des installations hydrauliques et de drainage et surtout intégrer la mise en œuvre d'une surveillance adaptée permettant d'alerter et d'intervenir pour arrêter le cas échéant les circulations. Dans ce contexte, plusieurs programmes ont vu le jour afin de réfléchir sur les meilleures façons d'intégrer ces risques à l'amont des projets, en phase de conception, et également sur les façons de le gérer sur le patrimoine existant que ce soit pour les lignes à grande vitesse [Nivon \& Pams Capoccioni, 2011] ou pour les lignes classiques [Lambert \& Le Noc, 2011]. Ils visent le développement de méthode d'analyses des risques ferroviaires liés à l'eau pour une meilleure anticipation des incidents. 
Le projet IRIP Rail a été lancé au sein de SNCF Infrastructure dans l'objectif d'améliorer la prise en compte du ruissellement dans les démarches existantes d'analyse des risques. Pour répondre à cet objectif, le logiciel en cours de développement au sein de SNCF depuis mai 2012 sur la base de la méthode IRIP proposée par Irstea intègre les exigences utilisateurs pour une application aussi bien en ingénierie qu'en recherche. Ce logiciel représente par ailleurs une première étape dans le développement d'applications opérationnelles à destination des agents de maintenance et de la politique de gestion du patrimoine en lien avec les des risques liés au ruissellement au sein de SNCF.

\section{PRÉSENTATION DE LA METHODE IRIP}

La méthode IRIP vise à calculer des Indicateurs du Ruissellement Intense Pluvial [Dehotin \& Breil, 2011a, $2011 b, 2012]$. Elle est basée sur une analyse de la dynamique des écoulements à partir de différentes couches d'informations géographiques : topographie, géologie, pédologie, occupation du sol et structures linéaires artificielles. Le traitement géomatique de ces données de base permet de calculer des indicateurs favorables à l'une des trois étapes hydrologique suivante : (P)roduction, $(\mathrm{T})$ ransfert et $(\mathrm{A}) \mathrm{ccu}-$ mulation du ruissellement. Les territoires sont découpés en mailles carrées dont la taille dépend de la précision de l'information disponible.

La succession des étapes qui peuvent conduire à une inondation par ruissellement intense pluvial est illustrée ci-dessous (Figure 3). Il s'agit de détecter dans un territoire donné les zones les plus sensibles à la production, au transfert et à l'accumulation du ruissellement. Plusieurs autres enchaînements sont possibles entre la production du ruissellement et son transfert vers l'aval, notamment la diffusion ou la restitution au milieu avant les zones d'accumulation. Le résultat est une cartographie de la sensibilité d'un territoire au ruissellement, indépendamment des événements météorologiques. Ce résultat montre un zonage maximaliste des emprises potentielles de ruissellement. L'intégration de l'information pluviométrique aux zonages permettrait à la fois d'affiner ces zonages et de caractériser les apports en débits de ruissellement, pour un scénario pluviométrique donné. Cet aspect fait partie des perspectives et n'est pas intégré en tant que tel dans la méthode IRIP.

La méthode IRIP permet donc d'identifier :

1. des zones de production (zones contributives) où les conditions au sol permettent la formation d'une lame d'eau en surface ;

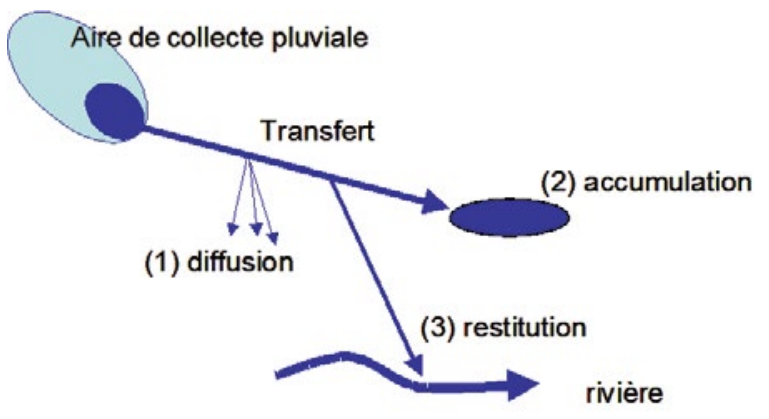

Figure 3 : Les étapes hydrologiques pouvant conduire à l'inondation par Ruissellement Intense Pluvial (RIP).
2. des axes de transfert naturels ou artificiels qui interceptent et redirigent l'écoulement du ruissellement vers l'aval ;

3. des zones d'accumulation des eaux de ruissellement, soit derrière un obstacle, soit par ralentissement quand la pente est moins forte, soit encore par stockage dans une dépression. Les accumulations sont conditionnées par l'importance des aires de contribution et l'existence des axes de transfert. La cartographie résultante restitue un aléa spatial.

Des applications ont été réalisées sur des bassins allant de quelques $\mathrm{km}^{2}$ à quelques milliers de $\mathrm{km}^{2}$, avec des données de précisions diverses [Dehotin et Breil, 2011a; Dehotin et Breil, 2011b; Arnaud et Dehotin, 2011; Bonnet, 2012]. L'évaluation des résultats a reposé elle aussi sur différentes sources d'informations à différentes échelles comme la base de donnés des arrêtés de catastrophes naturelles (http://www. catnat.net), une base de données de dysfonctionnements sur le territoire du Grand Lyon. Elle repose aussi sur la confirmation des processus physiques des étapes du ruissellement à partir d'un suivi métrologique sur un petit basin versant expérimental [de Lavenne, 2010; Dehotin \& Breil, 2012]. La méthode IRIP appliquée sur de grands territoires, avec des données à moyenne résolution (maillage kilométrique pour la topographie) permet un premier niveau de détection des zones où le processus de ruissellement est le plus probable. Une analyse plus fine de ces zones en regard des enjeux actuels ou à venir permet de poser un diagnostic opérationnel. Le recours à des données plus précises est alors indispensable.

Les trois cartes suivantes illustrent les résultats produits par les différentes étapes de l'analyse géomatique développée dans la méthode IRIP. Elles ont été réalisées sur un bassin périurbain de $6.7 \mathrm{~km}^{2}$ avec des données topographiques fines de type lidar (mailles de $2 \times 2 \mathrm{~m}$ ). Les données d'occupation des sols sont issues d'analyses d'images à haute résolution spatiale [Jacqueminet et al., 2013] et les données sols proviennent de campagnes de mesure directement sur le terrain en complément aux informations de la base de données des sols du programme IGCS [Gonzalez-Sosa et al., 2010].

La carte de la Figure 4a représente les niveaux de sensibilité des différentes parties du bassin à produire du ruissellement. On distingue des nuances de couleurs dans les versants avec les fortes sensibilités sur les plateaux et sur le réseau routier qui apparaît sous forme linéaire dans le haut bassin forestier. Il faut cependant noter aussi l'existence de zones à fort potentiel de production sur les bordures au nord et sud en haut de versant. Ces zones seront problématiques si leurs axes de transfert recoupent ou aboutissent dans les zones urbaines ou axes routiers qui sont situés juste en aval.

La carte de la Figure $4 \mathrm{~b}$ identifie les axes de transfert, sous condition d'existence d'une connexion à une aire de production. Le niveau de probabilité d'un axe de transfert dépend de ses caractéristiques locales propres et de celui de l'aire de production qu'il draine. On observe que les très fortes sensibilités concernent logiquement le réseau hydrographique mais aussi les zones de production situées en haut de versant dans la carte précédente.

La carte de la Figure 4c illustre les zones d'accumulation. On retrouve les deux zones de haut de versant nord et sud avec des sensibilités à l'accumulation allant de très forte (cas de la zone nord) à moyenne (zone sud). Elles peuvent être aussi des zones de production en absence de transfert ou dans des zones où le drainage est limité. C'est la situation des versants concaves qui produisent et accumulent le ruissellement.

La logique de la cartographie du ruissellement par la méthode IRIP permet d'aborder des réponses opérationnelles. Ainsi cette méthode ouvre des perspectives intéressantes 

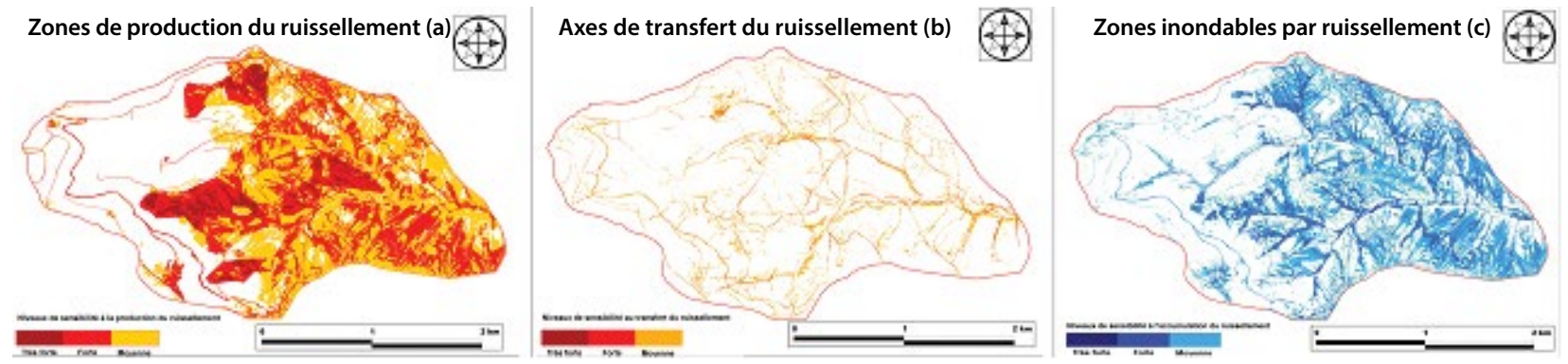

Figure 4 : cartographie par la méthode IRIP des zones de production (a) des zones de transfert (b) et des zones d'accumulation (c) - Source Irstea.

pour la gestion préventive des espaces pour limiter les risques liés au ruissellement. Cela peut consister à orienter les politiques pour :

- Ne pas aggraver le ruissellement des zones de production, de favoriser dans ces zones des techniques d'infiltration et de rétention en aidant à mieux choisir les sites à aménager ;

- Dégager ou réorienter les axes de transfert en évitant de barrer les chemins des eaux avec des aménagements linéaires (voiries, chemins de fer etc.) ou en déviant volontairement vers des zones d'étalement du ruissellement ;

- Limiter la vulnérabilité dans les lieux d'accumulation des eaux, par des politiques d'usage des sols et/ou de re-localisation d'enjeux.

\section{EXEMPLE DE MISE EN OEUVRE DE LA METHODE IRIP DANS LE CONTEXTE FERROVIAIRE}

Une première application de la méthode IRIP dans le contexte ferroviaire a été réalisée sur le tronçon Bayonne - Hendaye de la ligne Bordeaux - Irun. Ce tronçon de $36 \mathrm{~km}$ a fait l'objet d'une analyse des risques ferroviaires liés à l'eau en 2012 pour le compte de Réseau Ferré de France. Les résultats présentés ci-dessous ont été obtenus dans le cadre du projet de recherche IRIP Rail. Les clefs d'interprétation n'étant pas encore définies, ils ne peuvent pas faire l'objet, en l'état, d'un usage dans un cadre opérationnel d'analyse de risques.

Cette première application est une première étape de validation dans le contexte ferroviaire qui s'appuie sur les données du patrimoine ferroviaire existant, à savoir les incidents et les dispositifs hydrauliques, et les données géographiques disponibles dans le commerce. Cette première application est également une première étape de test et d'intégration de l'approche IRIP dans les processus d'analyse de risques ferroviaires qui s'appuie sur les méthodes existantes, les besoins utilisateurs (données d'entrée, environnement informatique) et les applications envisagées.

\section{IV.2. Eléments de validation de la méthode IRIP pour l'identification des sites ferroviaires soumis au ruissellement}

\section{IV.1.1. Objectif et enjeux}

Dans le cadre de l'identification des sites soumis au ruissellement, nous nous intéressons aux axes d'écoulement (ou zones de transfert) et à la submersion (ou zones d'accumulation). Cette première étape de validation de la méthode IRIP dans le contexte ferroviaire consiste à confronter les résultats de la cartographie du ruissellement obtenus par cette méthode aux données du patrimoine ferroviaire, à savoir les incidents recensés et les dispositifs hydrauliques existants, pour vérifier s'il existe une corrélation.

Une première application s'appuie sur les données topographiques à 30 mètres (données Intermap). Ils sont confrontés avec la base de donnée des incidents ferroviaires afin d'analyser la cohérence entre la présente de zones sensibles au ruissellement et les incidents. L'objectif de la démarche est d'identifier à l'échelle de ce tronçon les axes d'écoulement et les zones d'accumulation les plus importants et de les confronter aux données relatives aux fréquences des incidents.

Une deuxième application a été réalisée avec des données topographiques plus fines, résolution de 5 mètres sur un site particulier de la ligne étudiée. L'objectif est d'affiner la cartographie des zones d'aléa pour analyser plus finement un site et reconstituer le cheminement des écoulements. Sur la base d'un exemple, on montrera le rôle que peuvent jouer les dispositifs hydrauliques. Pour l'application avec des données topographiques à $5 \mathrm{~m}$, un petit tronçon de ligne faisant souvent l'objet d'incidents a été choisi.

Un premier enjeu est l'évaluation de la pertinence des données géographiques facilement accessibles. Notamment, les données topographiques issues de la base de données de la société Intermap ( 5 mètres et à 30 mètres de résolution). Les données d'occupation des sols utilisées sont les données Corine Land Cover 2006 à 100 mètres et les données sur la nature des sols sont issues de la base de données géographique des sols de France de l'INRA au 1/1.000.000.

Un deuxième enjeu est d'évaluer la disponibilité des informations de terrain sur l'environnement et le patrimoine ferroviaire permettant une confrontation pertinente. Les données du patrimoine ferroviaire considérées sont le tracé des voies ferrées, les incidents recensés sur le tronçon de ligne Bayonne-Hendaye de 1958 à 2012 ainsi que les dispositifs hydrauliques connus à ce jour. Une couche géomatique ferroviaire fournit le tracé des lignes ferroviaires. Les incidents sont recensés dans une base de données numérisée alimentée depuis 1998 en fonction des retours depuis le terrain. Cette base de données, malgré sa grande richesse et le fait qu'elle couvre toute la France métropolitaine n'est pas exhaustive; elle n'intègre pas forcément par exemple des incidents de faible gravité ou qui n'ont pas laissé de traces sur le site dans la mesure où ils ne sont pas signalés à la Direction de l'Ingénierie. Ainsi, il serait impossible 
de tirer des conclusions absolues sur des sites où ne figure aucun incident. Tous les dispositifs hydrauliques ne font pas l'objet de base de données nationale. Dans le cas présent, cette information est obtenue à partir d'un synoptique papier retranscrit en une couche géomatique exploitable. Les données relatives à l'environnement sont issues des bases de données de l'IGN, à savoir les cours d'eau et les routes.

\section{IV.1.2. Présentation des résultats de la cartographie du ruissellement}

La carte de ruissellement résultante de la méthode IRIP pour les données topographiques à 30 mètres est présentée sur la Figure 5. On note que les secteurs les plus sensibles au ruissellement (zones d'écoulement signalées en rouge et d'accumulation en bleu) sont situés au sud de Saint-Pierre-d'Irube, au niveau de la gare de Biarritz, et de la Gare de Bidart, dans la zone entre la gare de Guéthary et celle de Saint-Jean de Luz (secteur du cours d'eau de Basarun Erreka), la zone de la gare de Saint-Jean de Luz et la zone au nord de Hendaye.

La comparaison entre les zones d'aléas identifiées par la cartographie du ruissellement et les données d'incidents liées au ruissellement survenu sur la ligne est présentée sur la Figure 5. Les données d'incidents ont été analysées en utilisant les fiches descriptives pour ne retenir que les incidents effectivement liés au ruissellement. De manière générale, on note que 16 incidents survenus sur la ligne étudiée se trouvent soit sur ou à proximité immédiate d'un axe de transfert ou d'une zone d'accumulation de ruissellement, identifié par la cartographie avec un niveau de sensibilité moyen (3 sur une échelle de 5). Ces résultats illustrent une très bonne corrélation entre les incidents observés et les sites sensibles au ruissellement cartographiés par la méthode IRIP. Ces résultats vont dans le même sens que d'autres études réalisées par Irstea. Il faut cependant noter que les secteurs où des incidents liés aux coulées de boues sont signalées sont des zones où les axes de transfert et les zones d'accumulation ne présentent pas les potentiels de ruissellement les plus élevés. Les zones les plus sensibles identifiées par la cartographie ne sont pas systématiquement l'objet d'incidents relevés. Ceci peut être du à plusieurs raisons : soit que la cartographie réalisée est «pessimiste », soit que des incidents ne sont pas mentionnés dans la base de données que nous avons utilisée, soit que les ouvrages hydrauliques ont bien joué leur dans ces zones de la ligne étudiée. Il est difficile de conclure de manière objective sur ce dernier point. Toutefois, la densité des ouvrages de traversée présents dans ces zones (Figure 6a) montre la répartition des ouvrages hydrauliques de traversée qui assurent la transparence hydraulique de l'infrastructure ferroviaire et témoigne de l'importance des dispositifs hydrauliques pour le bon fonctionnement de l'infrastructure.

Les incidents ne sont pas forcément situés au droit du croisement dans l'espace entre l'aléa spatial et l'infrastructure. Certains incidents situés hors des zones d'aléa cartographiées peuvent très bien avoir un lien avec ces aléas, en raison du rôle que peuvent jouer les dispositifs hydrauliques et la géométrie de la voie dans le transfert des eaux de ruissellement dans l'emprise même du réseau ferroviaire. Cette situation, rencontrée sur ce tronçon, illustre bien le rôle des cartes d'aléa dont le croisement avec la vulnérabilité est nécessaire à l'établissement des cartes de risque. La définition de la vulnérabilité dans le contexte ferroviaire est complexe et nécessité d'intégrer le rôle des ouvrages hydrauliques qui confèrent indéniablement une certaine résilience. Les figures $6 \mathrm{a}$ et $6 \mathrm{c}$ montrent aussi que les incidents observés sont situés sur des secteurs sans ouvrage hydraulique de traversée et sur des axes de ruissellement " secondaires ». Une analyse avec des données plus fines est nécessaire afin de confirmer ce résultat des cartes d'aléa, en comparant de manière précise des axes de ruissellement et la localisation

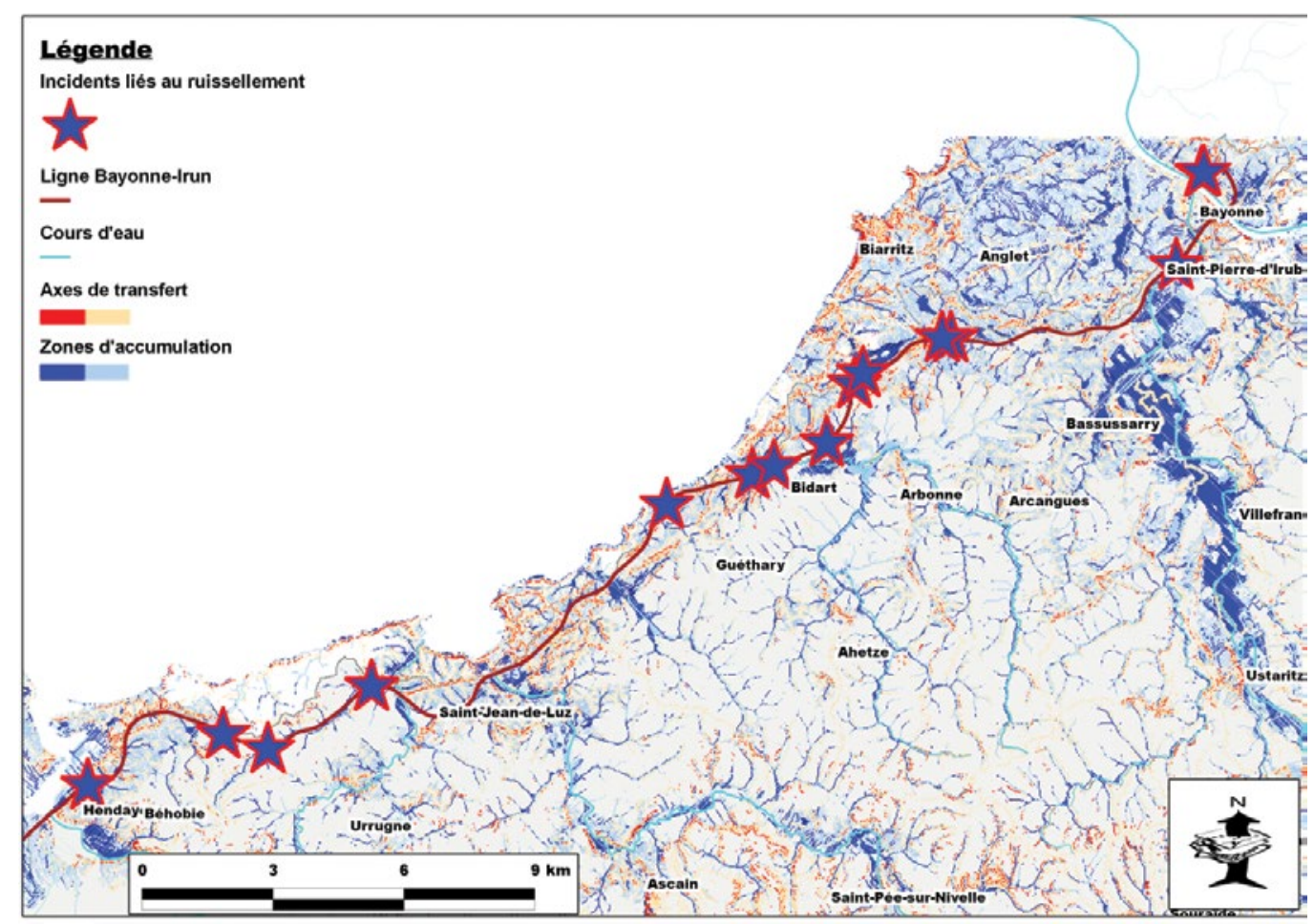

Figure 5 : Répartition des incidents recensés, toute gravité confondue, sur la ligne Bayonne-Irun. 


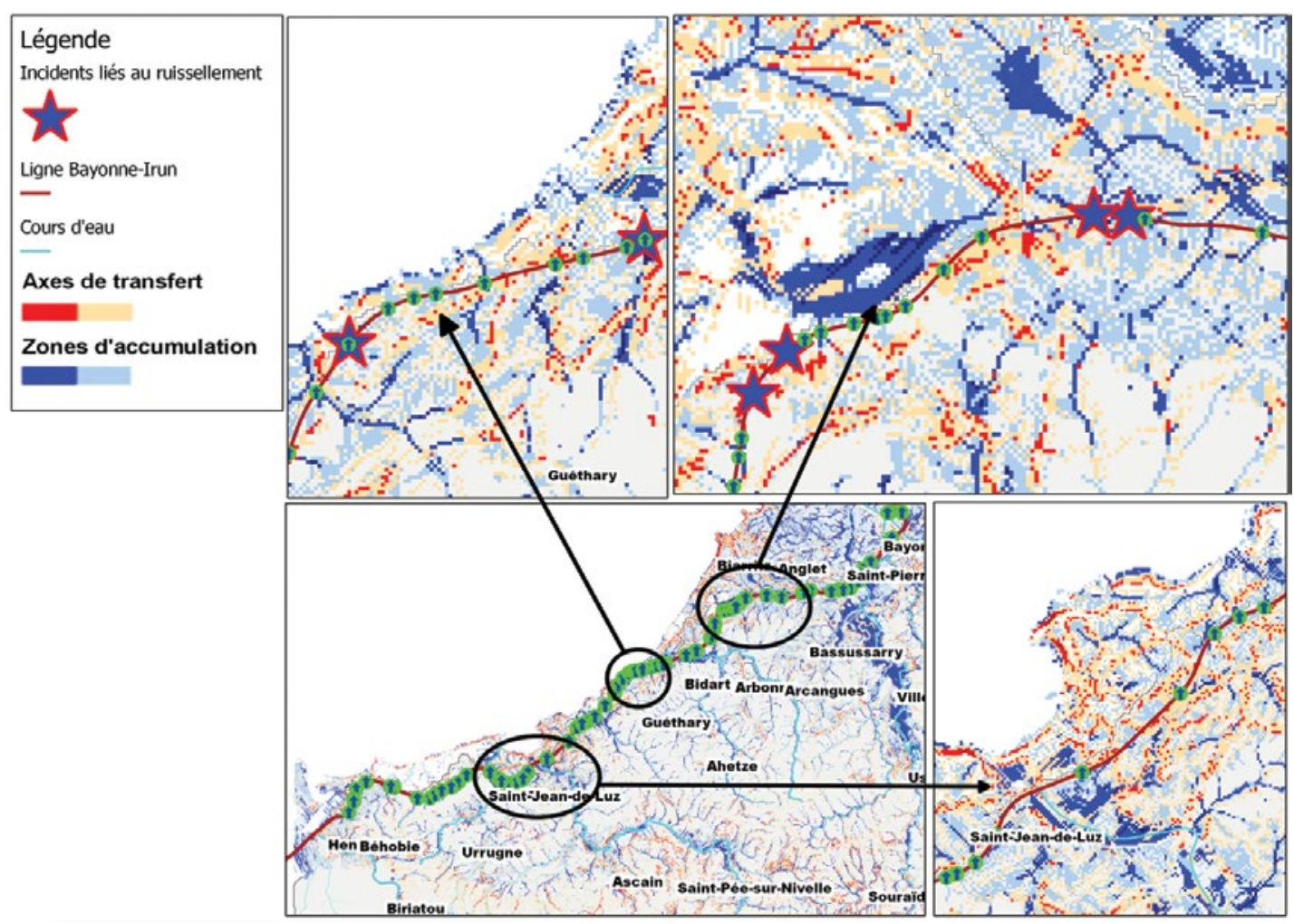

Figure 6 : Répartition des ouvrages hydrauliques de traversée sur la ligne Bayonne Irun.

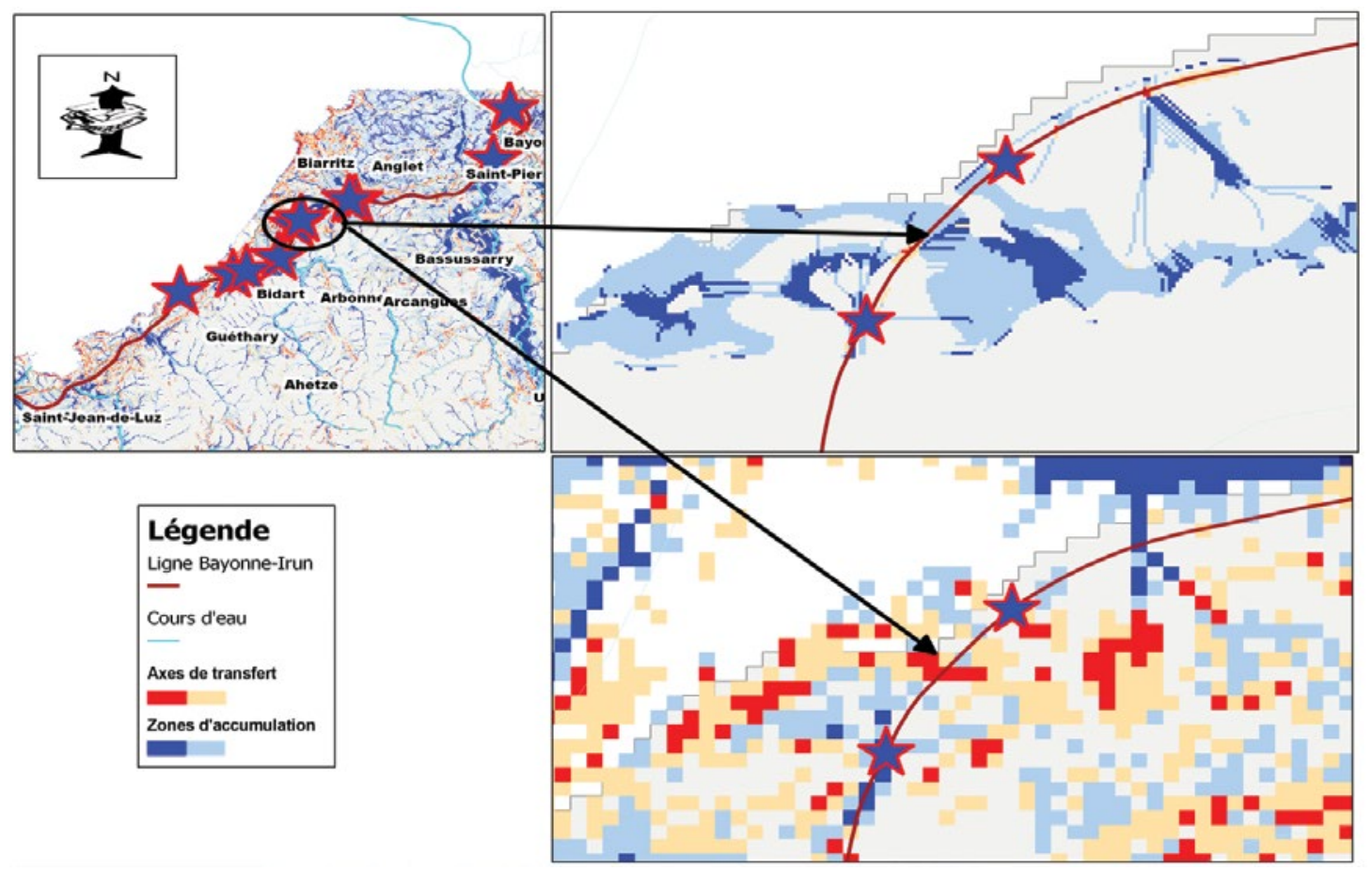

Figure 7 : Cartographie des axes de transfert et zones d'accumulation avec des données topographiques fines.

des ouvrages hydrauliques. Cette analyse à une échelle plus locale permettrait d'analyser finement sur quelques sites les désordres pour identifier ce qui relève d'un ruissellement exceptionnel et de la vulnérabilité du site.

Des données topographiques plus fines, maillage de $5 \mathrm{~m}$, ont été utilisées pour affiner la cartographie de l'aléa sur un site particulier de la ligne étudiée, souvent soumis à des incidents. Les résultats sont présentés sur la figure 7 . La délimitation des axes de transfert et des zones d'accumulation y est bien plus nette et plus adaptée à une utilisation locale. Les informations sur la géométrie de la voie (profil en long), en l'absence de drainage longitudinal, permettent d'expliciter le cheminement probable des eaux de ruissellement. 


\section{IV.2. Test et intégration de la méthode IRIP dans les démarches d'analyse des risques ferroviaires}

Au delà des objectifs de contribution à l'évaluation de la méthode IRIP, cette première application permet de tester en conditions réelles d'ingénierie la méthode et d'œuvrer pour son intégration dans ce contexte. Les développements informatiques du logiciel IRIP s'inscrivent dans ce cadre général.

La phase d'intégration, souvent sous-estimée dans les projets de recherche et développement, est une étape cruciale pour l'appropriation et l'utilisation effective de la méthode par les utilisateurs potentiels. Elle revêt des aspects aussi divers que :

— L'appropriation de la méthode par les utilisateurs potentiels

La méthode IRIP est une méthode innovante issue de la recherche nécessitant un transfert en conditions opérationnelles. Les cartes résultantes de son application offrent une approche nouvelle dans la compréhension et l'appréciation du ruissellement. Elle permet d'appréhender la complexité $\mathrm{du}$ phénomène contrairement aux approches actuellement disponibles. Il s'agit ici d'accompagner les utilisateurs futurs dans l'appropriation de cette approche et des résultats disponibles pour qu'ils l'intègrent dans leur pratique quotidienne. Ceci passe par des discussions approfondies sur la méthodologie et un accompagnement à partir de cas concrets d'application proposés par les utilisateurs. Ce transfert passe également par une adaptation de l'outil IRIP en développement au contexte d'ingénierie (données, systèmes d'information, procédures etc.).

Les résultats présentés précédemment sont une première étape dans la définition des informations requises en fonction de l'utilisation recherchée : Quelles données pour quelle utilisation ? Les données à maille grossière sont adaptées pour une analyse sur de grandes étendues spatiales pour identifier les sites les plus sensibles; les données à maille serrée sont préférables pour une vision locale du cheminement des eaux à l'échelle d'un site. Les données relatives au patrimoine complètent quant à elles la cartographie de l'aléa en apportant une approche de la vulnérabilité.

- La fourniture de conditions optimales d'utilisation et l'insertion dans l'environnement informatique

Les besoins d'applications de la méthode émergent des discussions avec les utilisateurs. Un besoin majeur est la simplification maximale de l'accès aux données existantes et de leurs traitements. L'accès aux données concerne l'obtention de données et les conditions d'utilisation. Il s'agit d'une part de fournir un environnement de travail compatible avec les données accessibles. Pour répondre aux enjeux liés aux traitements de données, SNCF a choisi d'intégrer dans l'outil IRIP un grand nombre de fonctionnalités de traitements de données géographiques. Le logiciel offre donc une interface définie en fonction des utilisations futures et un module de traitement de données géographiques.

Face au besoin de poursuivre l'amélioration de la méthode dans le cadre des actions de recherche, des modules ont été conçus de manière à permettre de modifier l'ensemble des paramètres intervenant dans le processus de cartographie définie à ce jour.

La pérennité du logiciel passe par une compatibilité avec l'environnement informatique existant (capacité de calcul des unités, conformité aux règles de développement) pour une meilleure prise en compte de sa maintenance (édition de guides de code, choix du code développement, définition d'un administrateur informatique et d'un administrateur métier). Pour cela une architecture souple modulaire et adaptative a été utilisée pour le développement du logiciel.

- Le développement de fonctionnalités compatibles avec les données d'entrée disponibles actuellement et dans le futur

A l'heure actuelle, les techniques d'acquisition de données évoluent rapidement grâce à des technologies telles que la photogrammétrie ou la lasergrammétrie, dont en particulier le lidar. Dans cette perspective, les modules de traitements de données implémentés ont du se rendre compatibles avec ces sources de données qui ont vocation à être utilisées de manière intensive dans les années à venir.

\section{PERSPECTIVES POUR LA GESTION DES RISQUES LIÉS AU RUISSELLEMENT}

SNCF a choisi de créer un logiciel à partir de la méthode IRIP car celle-ci présente un compromis intéressant en termes de données d'entrée nécessaires, de rapidité de traitement et de qualité des résultats pour des visées d'ingénierie et de gestion opérationnelle d'un réseau d'infrastructure de transport. Les premiers résultats montrent que ce logiciel peut appuyer l'expert pour la compréhension des phénomènes. En effet, la vision globale du bassin versant et des écoulements qui s'y déroulent complète sa connaissance du terrain.

La création de ce logiciel ouvre des perspectives d'applications diverses selon les enjeux de diverses parties prenantes du réseau ferré avec un seul objectif : améliorer la résilience du réseau ferré (sécurité et régularité des circulations ferroviaires) aux risques liés au ruissellement :

- Aider l'expert dans l'évaluation des impacts du ruissellement sur le système et la définition des actions à mettre en œuvre le cas échéant ;

- Appuyer l'ingénierie projet à mieux intégrer les risques liés à l'eau et à développer de nouvelles prestations ;

- Ouvrir la gestion des risques liés à l'eau au temps réel ;

- Proposer des outils d'aide pour la gestion des impacts aux tiers, y compris dans le cadre du changement climatique.

Outre l'aspect qualitatif, l'amélioration de l'évaluation des débits ruisselés à partir des informations produites par IRIP est en cours d'investigation au sein de SNCF. Plusieurs approches de calcul de débits sont en cours d'évaluation dont la formule rationnelle, la méthode de Crupédix et la modélisation de type débit-durée-fréquence ou méthode QdF [Galéa \& Ramez, 1995]. La méthode QdF a été largement validée par ailleurs depuis les années 80 sur des bassins ruraux et plus récemment sa dérivée $\mathrm{QdF}$ rapide sur des bassins à fort taux de ruissellement, vignoble de coteau, zones urbaines [Navratil et al. 2013].

Les travaux menés à SNCF œuvrent pour la fiabilité des résultats du logiciel tant pour les risques que pour les coûts et l'efficacité des ouvrages tout le long de leur cycle de vie.

Par ailleurs, des questions scientifiques restent à étudier afin de poursuivre l'amélioration de la méthode, nous citerons notamment :

- la question de la précision des données nécessaires à l'analyse pour une réponse adaptée, en lien avec les objectifs poursuivis ; - la question de la sensibilité des facteurs pris en compte dans l'analyse et éventuellement leur extension pour la prise en compte d'un plus grand nombre de configurations existantes dans le milieu naturel ;

- la question de la prise en compte du rôle joué par les réseaux anthropiques sur la dynamique du ruissellement; 
- la question de l'évaluation des débits de ruissellement dans les zones identifiées, notamment en contexte non jaugé - le rôle du ruissellement dans la genèse des coulées de boue et du transport solide.

Dans un contexte plus large que le ferroviaire, la méthode IRIP et le logiciel dérivé ouvrent des perspectives intéressantes pour anticiper les risques liés au ruissellement pour tout acteur du territoire en fonction de son échelle de considération et de ses objectifs. En effet, l'approche IRIP permet de travailler avec une vision globale de bassin versant, mais aussi à des échelles de tronçon d'infrastructure, de commune ou d'ouvrages ponctuels. Elle s'adapte à la précision des données d'entrée considérées selon les objectifs visés pour chaque étude. A des échelles locales (petites étendues spatiales), la méthode ouvre d'intéressantes perspectives sur la localisation des aménagements de rétention à l'amont des bassins, de protection contre les phénomènes d'érosion, etc. Sur de grands territoires, les zonages IRIP sont utilisables dans le cadre de la directive européenne sur les inondations, notamment sur le volet ruissellement, comme l'ont montré des applications réalisées pour le ministère chargé de l'environnement [Dehotin et Breil, 2011b].

Le logiciel en cours de développement sur la base de la méthode respecte les principes de modularité et d'évolutivité. Ceci permet aux intervenants du cycle de l'eau d'une part la possibilité d'en dériver des applications spécifiques selon leurs propres besoins, d'autre part de poursuivre les améliorations de la méthode dans le cadre d'actions de recherche.

\section{REFERENCES}

Arnaud P, Dehotin J. (2011) - Méthodes d'estimation de l'aléa ruissellement : Comparaison des méthodes SHYREG et IRIP. MEDD-Bureau des risques météorologiques. Irstea Publications

BonNET S. (2012) - Cartographie des zones potentielles de production ou d'accumulation du ruissellement de surface en région cévenole. Mémoire de stage de fin d'étude de Master II, Université de Montpellier. 2

Chazelle B., Lambert L., Pams Capoccioni C. (2014) - La vulnérabilité des infrastructures ferroviaires face aux évènements hydrologiques extrêmes. Etat des connaissances et gestion du risque. La Houille Blanche. 2 48-54

Dehotin J., Breil P. (2011) - Rapport technique du projet IRIP Cartographie de l'aléa ruissellement. Région Rhône-Alpes, Agence de l'Eau Rhône Méditerranée Corse. Irstea Publications

Dehotin J., Breil P. (2011) — Méthodes d'estimation de l'aléa ruissellement : Cartographie de l'aléa à échelle fine : Application de la méthode IRIP sur les bassins tests. MEDD-BRM. Irstea Publications

Dehotin J., Breil P. (2013) — Indicateurs du Ruissellement Intense Pluvial (IRIP): Détermination de l'aléa spatial inondation par ruissellement. Actes du colloque. Evénements extrêmes d'inondation, Lyon, 13-14 novembre 2013

De LaVenNe A. (2010) - Risque d'inondation par ruissellement: Instrumentation terrain et analyse géomatique. Memoire de fin d'étude, diplôme d'Ingénieur. Agrocampus Ouest-ESA

Galea G.; Ramez P. (1995) — Maîtrise du ruissellement et de l'érosion en coteau de vignoble : guide à l'usage des aménageurs. Cemagref Editions Référence Irstea : PUB00003156

Gonzalez-Sosa E., Braud I., Dehotin J., Lassabatere L., Angulo-Jaramillo R., Lagouy M., Branger F., Jacqueminet C., Kermadi S., Michel K. (2010) - Impact of land use on the hydraulic properties of the topsoil in a small French catchment. Hydrological Processes. 24(17) 2382-2399

Jacqueminet C., Kermadi S., Michel K., Beal D., Branger F., JANKOWSKY S., BRAUD I. (2013) - Land cover mapping using aerial and VHR satellite images for distributed hydrological modelling of periurban catchments: application to the Yzeron catchment (Lyon, France). Journal of Hydrology. 485 68-83

JoRf(1992) - Loi n ${ }^{\circ} 92-3$ du 3 janvier 1992 sur l'eau

JORF (2006) - LOI n $n^{\circ} 2006-1772$ du 30 décembre 2006 sur l'eau et les milieux aquatiques

Langlois P. Et Delahaye D. (2002) - RuiCells, automate cellulaire pour la simulation du ruissellement de surface. Revue Internationale de Géomatique. 12(4) 461-487

LAMBeRT L., Le NOC Y. (2011) - Evaluation des risques hydrauliques des voies ferrées : un exemple sur la ligne de Tarascon à Sète en région Languedoc Roussillon. Symposium International Georail

Le Bissonnais Y., Montier C., Daroussin J. And Kingd. (1998) - Cartographie de l'aléa Erosion des sols en France. IFEN Collection Etudes et Travaux. 18

Le Gouee P. Et Delahaye D. (2008) - Modélisation et cartographie de l'aléa érosion des sols et des espaces de ruissellement dans le Calvados.» Rapport d'étude

LrPC Et Cete NoRmandie. (2009) - Étude bibliographique de trois méthodologies appliquées au risque érosion. Synthèse et perspectives en vue d'une cartographie départementale du risque ruissellement «Laboratoire Central des Ponts et Chaussées

Navratil O., Breil P., Schmitt L., Grospretre L., Albert M.B. (2013) - Hydrogeomorphic adjustments of stream channels disturbed by urban runoff (Yzeron river basin, France). Journal of Hydrology. 485 24-36

Nivon D., Pams Capoccioni C. (2011) - Analyse du risque hydraulique sur lignes à grande vitesse exploitées. Symposium International Georail

Pams Capoccioni C., Cheetham M. (2012) — La transparence hydraulique d'infrastructures ferroviaires existantes suite aux inondations de novembre 1999. Actes Congrès SHF « Evènements extrêmes fluviaux et maritimes " 1-2 février 2012, Paris

Satin M. (2006) - Guide technique de l'assainissement 3e édition. Éd. le Moniteur. Ministère de l'Agriculture (1980-1982). Synthèse Nationale sur les crues des petits bassins versants. Fascicule 1 : éléments de pluviométrie ; fascicule 2 : la méthode SOCOSE ; fascicule 3 : la méthode CRUPEDIX : fascicule 4 : caractéristiques averses-crues. $726 \mathrm{p}$ 